\title{
Solvothermal synthesis of a polyaniline nanocomposite - a prospective biosensor electrode material
}

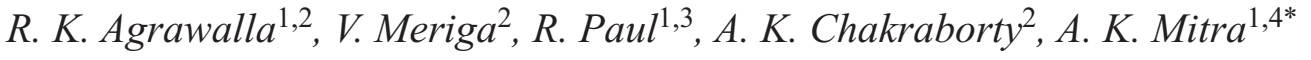 \\ ${ }^{1}$ Nanoscience Laboratory, Department of Physics, National Institute of Technology Durgapur, M. G. Avenue, 713209 \\ Durgapur, India \\ ${ }^{2}$ Carbon Nanotechnology Laboratory, Department of Physics, National Institute of Technology Durgapur, M.G. Avenue, \\ 713209 Durgapur, India \\ ${ }^{3}$ Presently D S Kothari Postdoctoral Fellow, Materials Engineering Department, Indian Institute of Science, 560012 \\ Bangalore, India \\ ${ }^{4}$ Presently Visiting Professor, National Institute of Technology, 713209 Durgapur, India
}

Received 14 February 2016; accepted in revised form 1 May 2016

\begin{abstract}
Polyaniline (PANI) is the most important conducting polymer with excellent electrochemical properties. So PANIbased biosensors may find wide applications in medical diagnostics. We report here a ternary nanocomposite of gold nanoparticle- decorated single- walled carbon nanotubes (SWCNTs) embedded in sulfonated polyaniline matrix, prepared using a simple solvothermal chemical route. The structural and morphological characteristics have been determined by electron microscopy, X-ray diffraction and Raman spectroscopy. Optical characteristics of the nanocomposite have been determined by ultraviolet (UV)-visible absorption spectroscopy and photoluminescence spectroscopy. The direct current (DC)-conductivity measurement of the material shows a significant increase in electrical conductivity at $353 \mathrm{~K}$ from $7.80 \cdot 10^{-2} \mathrm{~S} / \mathrm{m}$ for pure SPANI to $10.91 \mathrm{~S} / \mathrm{m}$ for the 3-phase nanocomposite as synthesized in the present investigations. Thus the incorporation of SWCNT/Au nanohybrid fibers in the PANI matrix enhanced its electrical properties. Sulfonation increased the processability of the material, as the samples have now been found to be soluble in water and common organic solvents like DMSO. Such a functional nanocomposite will make an excellent biosensor electrode material.
\end{abstract}

Keywords: nanocomposites, SWCNT/Au fibers, sulfonated polyaniline, biosensor

\section{Introduction}

Biosensors make a very important area of intensive investigation for their wide applications in medical diagnostics and other useful fields. Polyaniline (PANI) is an interesting polymer due to its conducting properties, relatively cheap price and good stability and hence is one of the most studied conducting polymers in recent years [1-3]. Moreover as polyaniline (PANI) has excellent electrochemical properties, it is an automatic choice in the design of sensor electrode materials.

\footnotetext{
*Corresponding author, e-mail: akmrecdgp@yahoo.com
} (C) BME-PT
Carbon nanotubes (CNTs) have attracted great research interest due to their many interesting properties leading to numerous potential applications [4]. In particular, functionalized SWCNTs, due to active functional groups on their surfaces are suitable for even wider range of applications such as in sensors, polymer composites etc. [5-7]. Consequently, researchers have combined functionalized CNTs with PANI $[8,9]$ and evaluated their properties. However, as PANI is insoluble in water and common organic solvents, in order to improve its processability, researchers often carry out sulfonation in which sul- 
fonic acid functional groups are attached to PANI and the product thus formed is a soluble polymer called sulfonated polyaniline (SPANI) as pointed out in their work by some investigators $[10,11]$.

One problem in this approach is that the electrical conductivity is greatly reduced by sulfonation which puts some limitation on the use of the polymer. Owing to their excellent electrical properties, it is expected that incorporating CNTs and in particular, metal nanoparticle-decorated CNTs, into SPANI matrix may result in composites having enhanced electrical properties due to synergetic influence, without compromising much the electrochemical properties of the polymer. Noble metal nanoparticles such as gold, silver etc. are special choice for decorating CNTs, in the design of biosensors, due to their high chemical stability and excellent electrical conductivity. Gold is particularly important because of its known affinity towards attachment with biomolecules, which is important for its application in biosensors.

However, in spite of the interest, till todate only few reports have been published on this topic. The earliest report is the one by Santhosh et al. [12] who prepared electrocatalysts by dispersing gold nanoparticles into a PANI grafted multi-walled carbon nanotube (MWCNT) matrix which can have application in direct methanol fuel cells. The PANI/gold ( $\mathrm{Au})$ composite hollow spheres were synthesized by Feng et al. [13], which showed more than three times higher electrical conductivity than that of pure PANI hollow spheres. The composite further showed enhanced electrocatalytic activity for the oxidation of dopamine when immobilized onto a carbon electrode. Wang et al. [14] synthesized PANI/MWCNT/Au composite film via a two-step electrochemical process, with $\mathrm{Au}$ nanoparticles dispersed in the PANI-MWCNT film. The composite exhibited good electrochemical and electrocatalytic activity. Chang et al. [15] fabricated $\mathrm{PANI} / \mathrm{Au} / \mathrm{MWCNT}$ nanocomposite by one pot synthesis when aniline molecules were adsorbed and polymerized on the surfaces of MWCNTs. The composite showed superior sensitivity in ammonia gas detection. Lee et al. [16] prepared composites of single-walled carbon nanotubes (SWCNTs), PANI and $\mathrm{Au}$ nanoparticles in an in-situ one-pot fashion, by using $\gamma$-radiation as source for initiation of polymerization and generation of Au nanoparticles. Guo and
Peng [17] synthesized SWCNT/PANI/Au composite by using aromatic amine chemistry, and found the material to be a good electrode material for use in electrochemical sensors. Rima and Mitra [18] prepared and studied the optical and electrical properties of SWCNT/Au nanohybrids, and suggested that the composite could be used as efficient catalyst in chemical industry, as well as in medical diagnostics. What we have observed is that these studies have mostly used PANI rather than SPANI and hence the synthesized material has very little processability. So the ternary composites containing SWCNT, SPANI and gold nanoparticles deserve greater research attention, and would lead to a better biosensor electrode coating material.

In an effort to understand the influence of gold nanoparticles on SWCNT/SPANI composites, we present a simple chemical synthesis of a composite containing sulfonated polyaniline (SPANI) and SWCNT/Au hybrid nanostructures. The gold nanoparticle- decorated SWCNTs (SWCNT/Au nanohybrid) were prepared by a simple wet chemical process. The morphology and structural properties of the nanocomposites thus produced were characterized by a number of analytical techniques including X-ray diffraction (XRD), scanning electron microscopy (SEM), high resolution transmission electron microscopy (HRTEM), Raman spectroscopy etc. Optical properties of the nanocomposites were evaluated by UV-visible absorption spectroscopy and photoluminescence (PL) spectroscopy. Further, the electrical properties were studied through DC-conductivity measurement.

\section{Materials and methods}

\subsection{Materials}

The SWCNTs used in this work were supplied by Chengdu Organic Chemicals Co. Ltd, P. R. China, prepared by electric arc discharge method. The average diameter, length and purity of the SWCNTs, as stated by the manufacturer were 1-2 nm, 1-3 $\mu \mathrm{m}$, and $95 \mathrm{wt} \%$, respectively. Aniline, 1,2-dichloroethane (DCE) and ammonium persulfate were supplied by Merck Specialties Pvt. Ltd., Mumbai, India. The chlorosulfonic acid and trisodium citrate used in our work were supplied by LOBA Chemie Pvt. Ltd., Mumbai, India. Except SWCNTs, all other chemicals were used as received without further purification. 


\subsection{Purification and functionalization of SWCNTs}

The as-received SWCNTs were purified following methods reported elsewhere [11]. In brief the purification involved heating in a muffle furnace at $350^{\circ} \mathrm{C}$ in air for $1 \mathrm{~h}$ followed by soaking and stirring in $6 \mathrm{M}$ $\mathrm{HCl}$ for $12 \mathrm{~h}$. The acid-treated SWCNTs were filtered and washed thoroughly with deionized water after which further treated in a mixture of concentrated $\mathrm{HNO}_{3} / \mathrm{H}_{2} \mathrm{SO}_{4}$ in 1:3 volume proportion for $4 \mathrm{~h}$ followed by washing with dilute $\mathrm{NaOH}$ aqueous solution and filtration until the $\mathrm{pH}$ became neutral. The product thus obtained was SWCNTs functionalized with carboxylic $(-\mathrm{COOH})$ acid groups (f-SWCNTs).

\subsection{Synthesis of SWCNT/Au nanohybrid}

Tetrachloroauric acid $(0.0142 \mathrm{~g})$ was added to deionized water $(50 \mathrm{~mL})$ and stirred at $100^{\circ} \mathrm{C}$ for $30 \mathrm{~min}$. In another beaker, trisodium citrate solution (reducing agent) was prepared by mixing it with de-ionized water and stirred at $100^{\circ} \mathrm{C}$ for $30 \mathrm{~min}$. SWCNTs $(0.0612 \mathrm{~g})$ was then added to the solution containing tetrachloroauric acid and simulataneously the citrate solution was also added into it followed by stirring at $80^{\circ} \mathrm{C}$ for further $30 \mathrm{~min}$. The resultant mixture was then filtered using vacuum filtration system (Millipore membrane, pore size $\sim 0.22 \mu \mathrm{m}$ ). The precipitate was then washed with deionized water and filtered again to obtain SWCNT/Au nanohybrid fibres. The sample was then dried under an infrared (IR) lamp.

\subsection{Synthesis of SPANI and SPANI/SWCNT composite}

The detailed method for the synthesis of sulfonated polyaniline (SPANI) has been reported elsewhere by our group [11]. In brief, this involved the use of aniline hydrochloride, ammonium persulfate and chlorosulfonic acid as precursors. SPANI/SWCNT composite containing $6 \mathrm{wt} . \%$ SWCNT (as estimated from TGA) was prepared by dissolving SPANI and SWCNT in water followed by filtration.

\subsection{Synthesis of SPANI/SWCNT/Au ternary composite}

SWCNT/Au nanohybrid (0.03 g) was added into deionized water followed by addition of SPANI aqueous solution and stirred for $3 \mathrm{hrs}$ at $60^{\circ} \mathrm{C}$. The SPANI/
SWCNT/Au composite was obtained by filtering the solution and drying the filtrate.

\subsection{Characterization methods}

The high resolution micrographs were recorded using a (Carl Zeiss) field emission scanning electron microscope (FESEM), and a high resolution transmission electron microscope (HRTEM) JEM-2010 (JEOL Japan) with operating acceleration voltage of $200 \mathrm{kV}$. Raman spectroscopy was performed using EZ Raman-M field portable Raman analyzer (Enwave Optronics Inc., USA), using a diode laser of wavelength $785 \mathrm{~nm}$ as excitation source. The thermogravimetric analysis (TGA) was carried out with Perkin Elmer Pyris-1 TGA thermogravimetric analyzer (USA) at a heating rate of $10^{\circ} \mathrm{C} / \mathrm{min}$ in nitrogen atmosphere. The optical absorbance spectra were recorded using a U-3010 UV-visible absorption spectrophotometer (HITACHI, JAPAN). Photoluminescence spectra of the samples were acquired using a PerkinElmer LS-55 Fluorescence Spectrophotometer (USA). The electrical conductivity of the samples was measured in ambient atmosphere by a FourProbe set-up (DFP-02, Scientific Equipment, India).

\section{Results and discussion}

The morphology of SPANI/f-SWCNT/Au composite was investigated by electron microscopy. The FESEM micrographs of the sample are shown in Figure 1a, in which we observe gold nanoparticles attached to the surfaces of nanotube bundles. The presence of gold is confirmed in the energy dispersive analysis by X-ray spectroscopy (EDAX) spectrum shown in Figure $1 \mathrm{~b}$. The presence of carbon comes from both f-SWCNT and SPANI. The morphology shows tubular structure of carbon nanotube bundles with attached gold nanoparticle clusters on its surfaces, and covered by polyailine matrix. Such structures have been observed by earlier investigators [19].

In the HRTEM image (Figure 2), we observe the presence of CNT bundle coated with SPANI and decorated with gold nanoparticles. The average diameter of tubular structure is measured to be about $\sim 12 \mathrm{~nm}$ from the HRTEM micrograph. The dark spots represent the gold nanoparticles with particle size in the range of 2 to $7 \mathrm{~nm}$.

The X-ray diffractograms of SPANI, f-SWCNT, SPANI/f-SWCNT binary composite $(6 \mathrm{wt} \%)$ and 


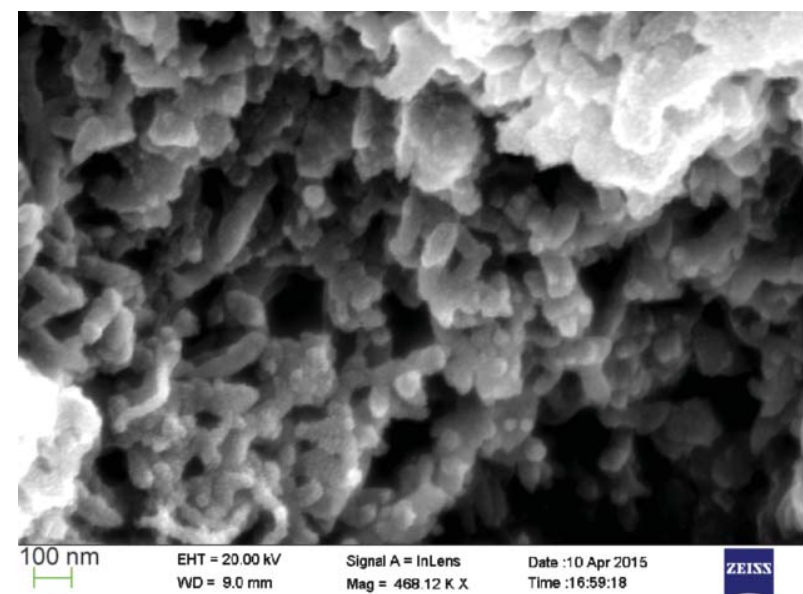

a)

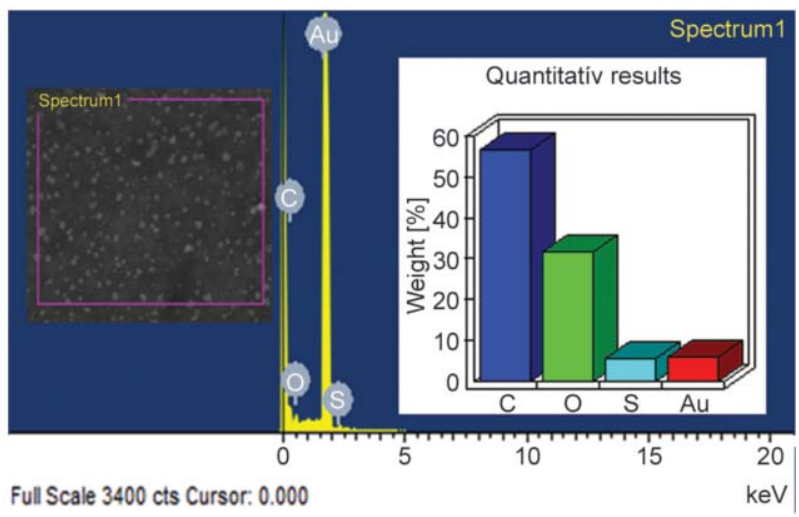

b)

Figure 1. (a). FESEM image of SPANI/f-SWCNT/Au ternary nanocomposite, (b) EDAX analysis of SPANI/f-SWCNT/Au ternary composite showing SEM picture in the left, spectrum in the middle and the quantity of elements in the right

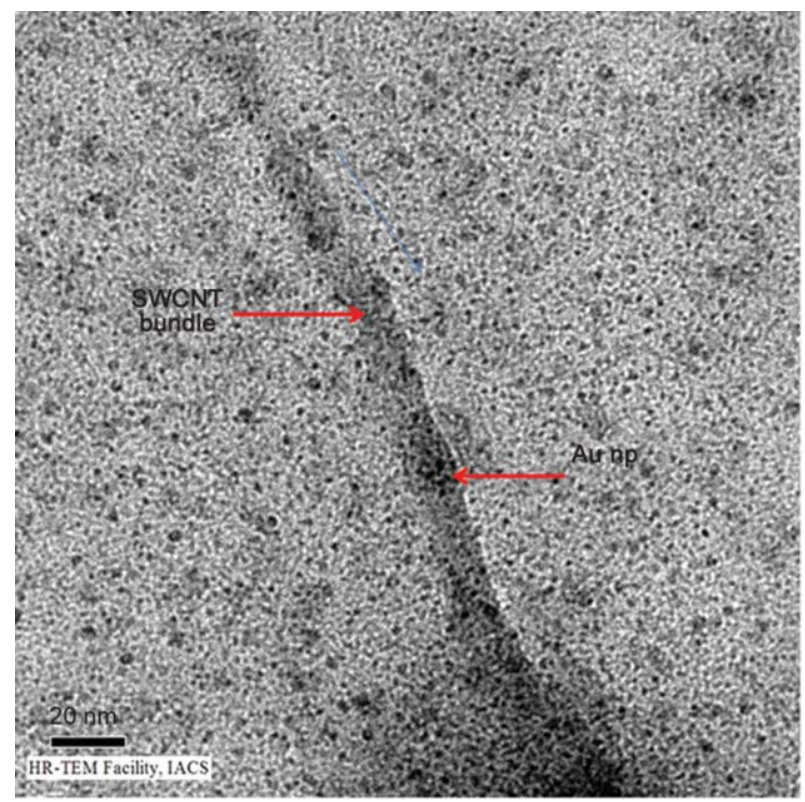

Figure 2. HRTEM micrograph of SPANI/f-SWCNT/Au ternary composite

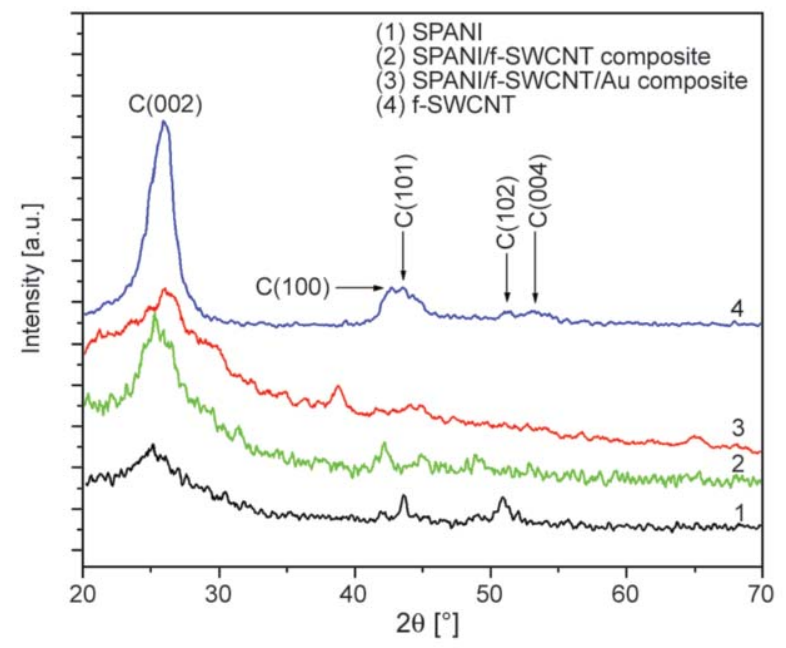

Figure 3. XRD patterns of SPANI, f-SWCNT, SPANI/ f-SWCNT and SPANI/f-SWCNT/Au
SPANI/f-SWCNT/Au ternary composite are compared in Figure 3. The features observed for SPANI and SWCNT (JCPDS card no. 75-1621) are in good agreement with the reported literature. The magnified diffractogram of the ternary composite is separately plotted in Figure 4, for better clarity in which the characteristic peaks of CNT and gold are clearly visible. The sharp peaks at $37.8,43.7$ and $64^{\circ}$ are assigned to (111), (200) and (220) planes of the reflections of face-centered cubic (fcc) structure of metallic Au nanocrystals (JCPDS card no. 00-004-0784). The average size of $\mathrm{Au}$ nanoparticles in the composite is calculated to be $\sim 8 \mathrm{~nm}$ using the DebyeScherrer formula. The general features of the diffractogram of SPANI are retained in the composite, but the crystallinity of Au and SWCNT manifest in the SPANI background.

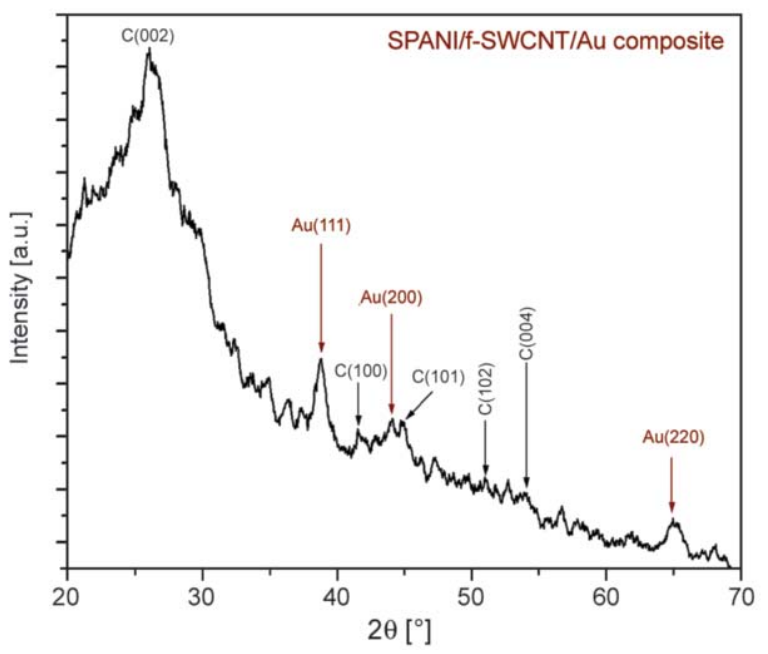

Figure 4. Magnified XRD pattern of SPANI/f-SWCNT/Au ternary composite 


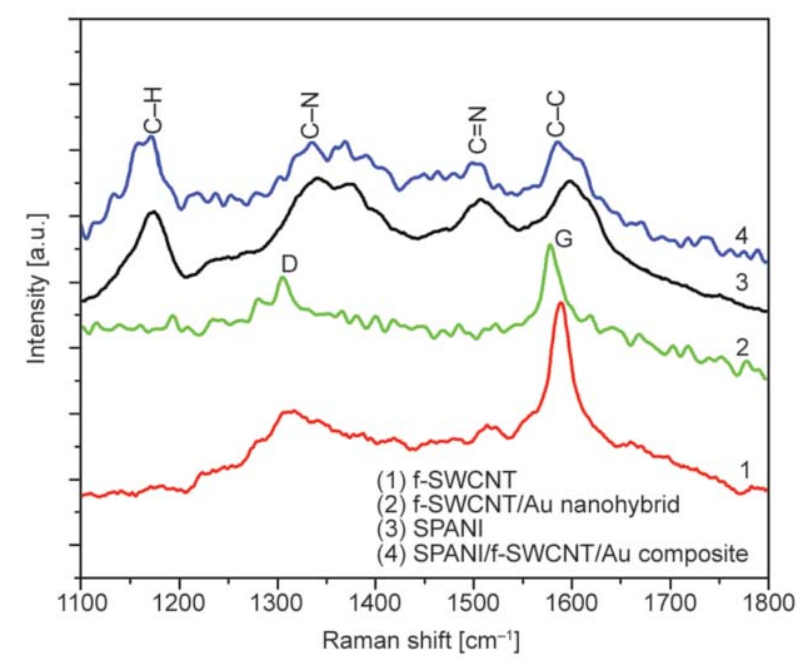

Figure 5. Raman spectra of SPANI, f-SWCNT, f-SWCNT/ Au hybrid and SPANI/f-SWCNT/Au ternary composite

Figure 5 shows the Raman spectra of SPANI, fSWCNT, f-SWCNT/Au nanohybrid and SPANI/fSWCNT/Au ternary composite. The spectra of fSWCNT (curve 1) and f-SWCNT/Au hybrid (curve 2) show peaks assigned to both G-band and D-bands of SWCNT. The position of the G-band of SWCNT is red-shifted from 1589 to $1577 \mathrm{~cm}$ on decorating with Au nanoparticles, indicating charge transfer between SWCNT bundles and Au nanoparticles [20, 21]. Further, the ratio of the intensity of these two peaks (I/I) increases from 0.51 to 0.79 on attaching gold nanoparticles on the SWCNT surfaces indicating an increased disorder and lowering of symmetry as expected. In the ternary composite (curve 4), the features are dominated by those of SPANI possibly due to thick coating of SWCNT/Au nanohybrids with SPANI polymer.

Figure 6 shows the TGA thermograms of f-SWCNT, SPANI and SPANI/f-SWCNT/Au composite. The thermogram of the ternary composite is similar to that of SPANI, which is due to very low nanohybrid content. While f-SWCNTs do not show any noticeable loss of mass until about $550{ }^{\circ} \mathrm{C}$, the SPANI and its composites show almost complete weight loss at a temperature of $580{ }^{\circ} \mathrm{C}$. This is not surprising given SPANI being a relatively soft polymer and that the ternary sample has very low CNT content. The interesting thing to observe here is that for SPANI/ f-SWCNT/Au composite, some residual weight (of about $7 \%$ ) is observed even at temperatures above $700{ }^{\circ} \mathrm{C}$. This is due to the undecomposed gold metal

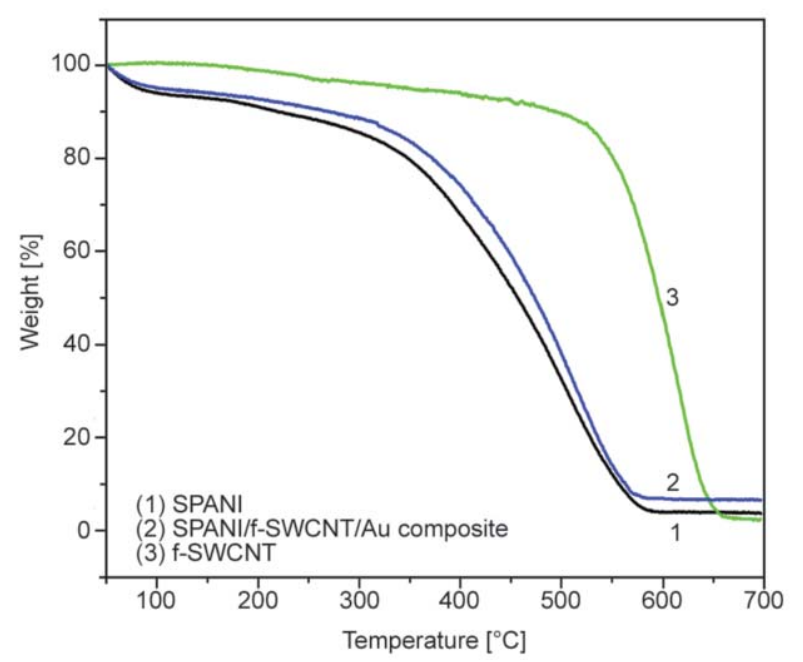

Figure 6. Thermograms of SPANI, f-SWCNT, and SPANI/ f-SWCNT/Au ternary composite

present in the nanocomposite, although the residual weight for pure SPANI is less than $4 \%$. Moreover SPANI may also have undergone some incomplete decomposition, as has been observed in some earlier investigations.

For optical spectroscopy, the samples were dissolved in an organic solvent dimethyl sulfoxide (DMSO) which is a good solvent for both SPANI and its SWCNT-based composites.

The UV-visible absorption spectra of different samples are compared in Figure 7. The spectrum of SPANI consists of humps at $355 \mathrm{~nm}$ ( $\pi-\pi^{*}$ transition), $455 \mathrm{~nm}$ (polaron- $\pi^{*}$ transition) and beyond $750 \mathrm{~nm}(\pi$-polaron transition). The different absorbance bands for SPANI/f-SWCNT binary composite are clearly evident. In the spectrum of SPANI/f-SWCNT/Au nanocomposite, there is a shift of the $\pi-\pi^{*}$ transition to

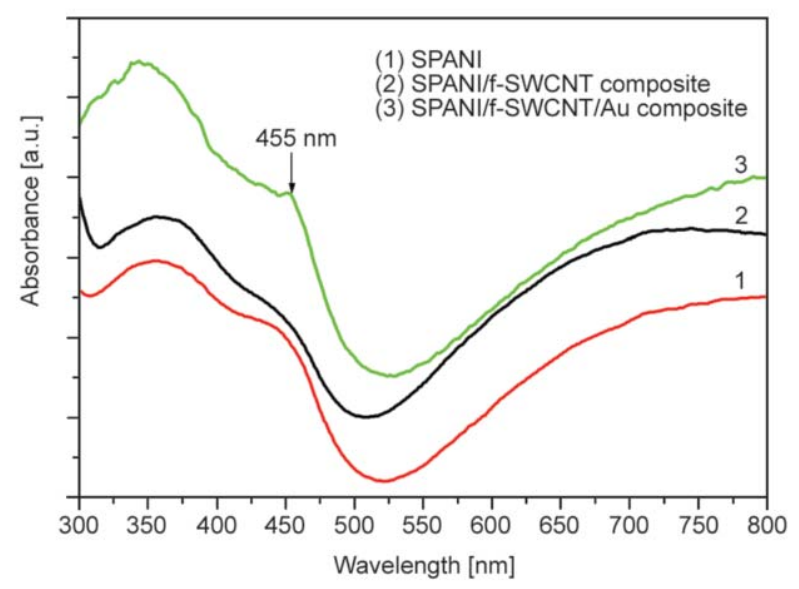

Figure 7. UV-vis absorbance spectra of SPANI, SPANI/ f-SWCNT binary composite and SPANI/f-SWCNT/ Au ternary composite 
$340 \mathrm{~nm}$. We also observe a shoulder peak at about $455 \mathrm{~nm}$, which may correspond to the modified SPR band of gold nanoparticles. The SPR band for gold nanoparticles is generally observed at about $520 \mathrm{~nm}$ which might have changed its position with reduced intensity. As SPANI provides a different dielectric medium to Au nanoparticles and as the SPR band is sensitive to its environment, shifting and quenching effects take place. There is a possibility of merging of the strong SPR band with the polaron band of SPANI, which is also observed in this region. Similar observations have been reported earlier [16].

The optical band gaps of the investigated samples are estimated using Tauc relation [22]. The optical band gap of pure SPANI is estimated to be $3.75 \mathrm{eV}$. For the samples of SPANI/f-SWCNT and SPANI/ $\mathrm{f}-\mathrm{SWCNT} / \mathrm{Au}$ composites, it is estimated to be 3.66 and $3.41 \mathrm{eV}$ respectively, as shown in Figure 8. The decrease in band gap with incorporation of SWCNT and SWCNT/Au hybrid is compatible with their better electrical conductivities [23].

The PL spectra for the different samples are obtained for an excitation wavelength of $300 \mathrm{~nm}$ and are shown in Figure 9. SPANI shows a hump around $445 \mathrm{~nm}$. There is a quenching effect of the broad emission band of SPANI upon interactions with CNT/Au nanohybrid, which generally takes place when the polymer is doped to a highly conductive state [24].

Figure 10 shows the de electrical conductivity of SPANI, SPANI/f-SWCNT binary composite and SPANI/f-SWCNT/Au ternary composite at temperatures ranging from 313 to $353 \mathrm{~K}$. We observe an in-

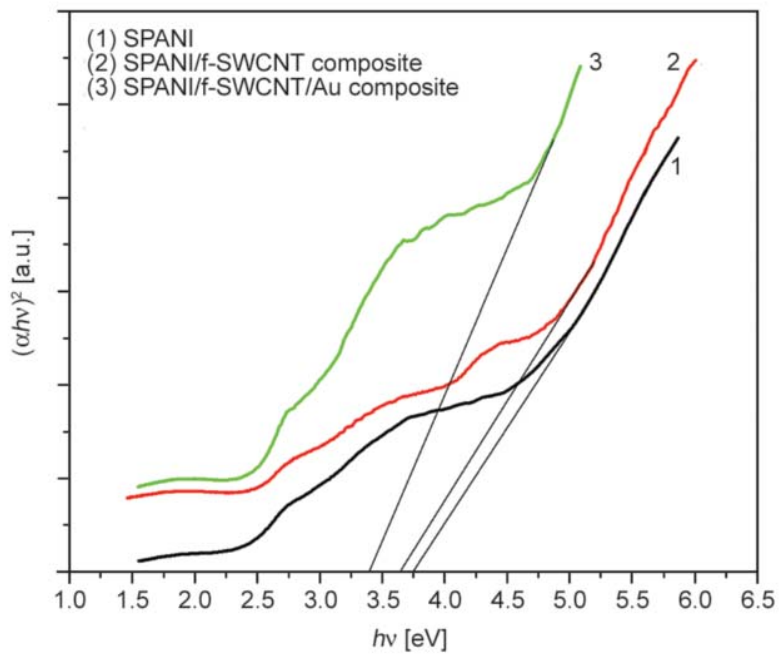

Figure 8. Plot of $(\alpha h v)^{2}$ vs. hv for SPANI, SPANI/f-SWCNT binary composite and SPANI/f-SWCNT/Au ternary composite for optical band gap calculation

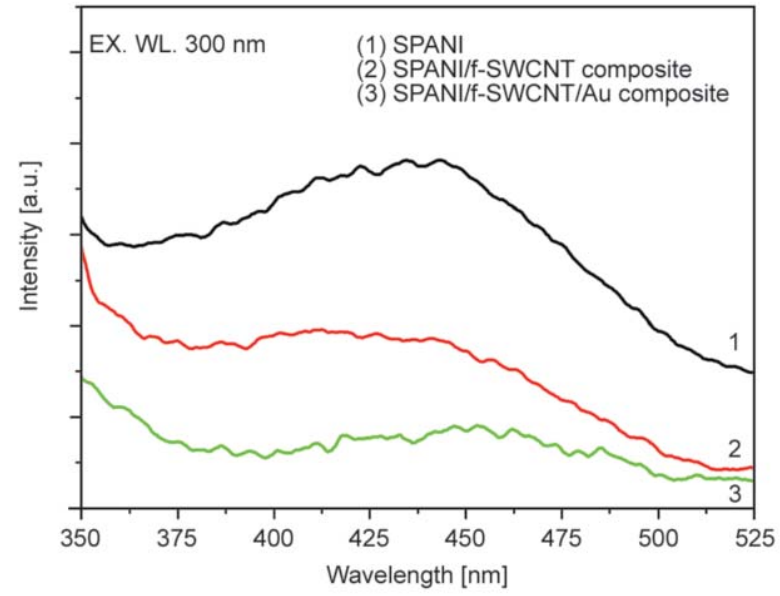

Figure 9. Photoluminescence spectra for SPANI, SPANI/fSWCNT binary composite and SPANI/f-SWCNT/ Au ternary composite

crease in the conductivity with increase in temperature for all these samples, thus showing semiconductor like behavior. On adding the nanotubes or the nanohybrids to the polymer, the conductivity increases. At $353 \mathrm{~K}$, the conductivity values for SPANI, SPANI/fSWCNT and SPANI/f-SWCNT/Au samples are $7.80 \cdot 10^{-2}, 5.58 \cdot 10^{-1}$ and $10.91 \mathrm{~S} / \mathrm{m}$, respectively. Therefore, the conductivity of the ternary composite becomes nearly 137 and 25 times higher than those of SPANI and SPANI/f-SWCNT binary composite respectively, although the conductivities still remain in the semiconducting range. This increase is attributed to the presence of gold nanoparticles, making available more electrons as charge carriers for the n-type

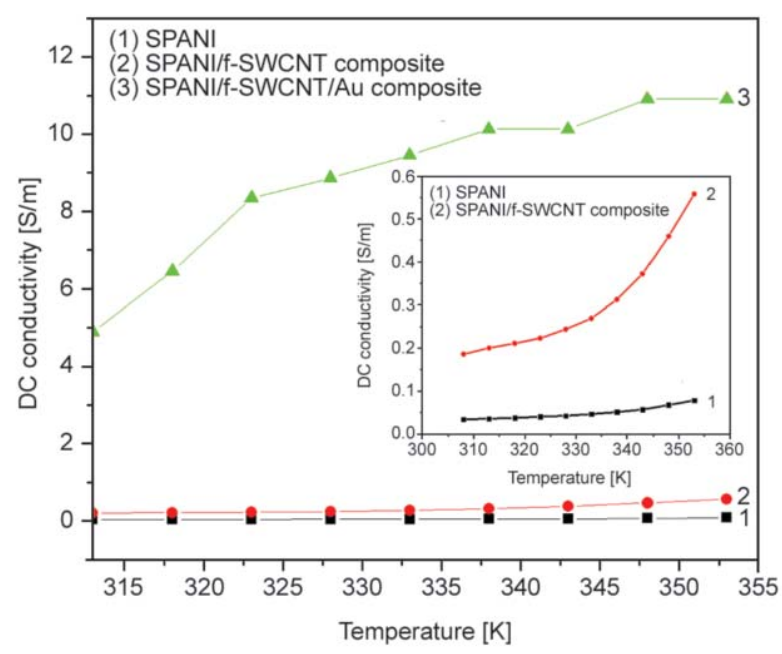

Figure 10. Comparison of dc conductivity of SPANI/ f-SWCNT/Au ternary composite with SPANI and SPANI/f-SWCNT binary composite at varying temperature; comparision between SPANI and SPANI/f-SWCNT is shown in the inset 


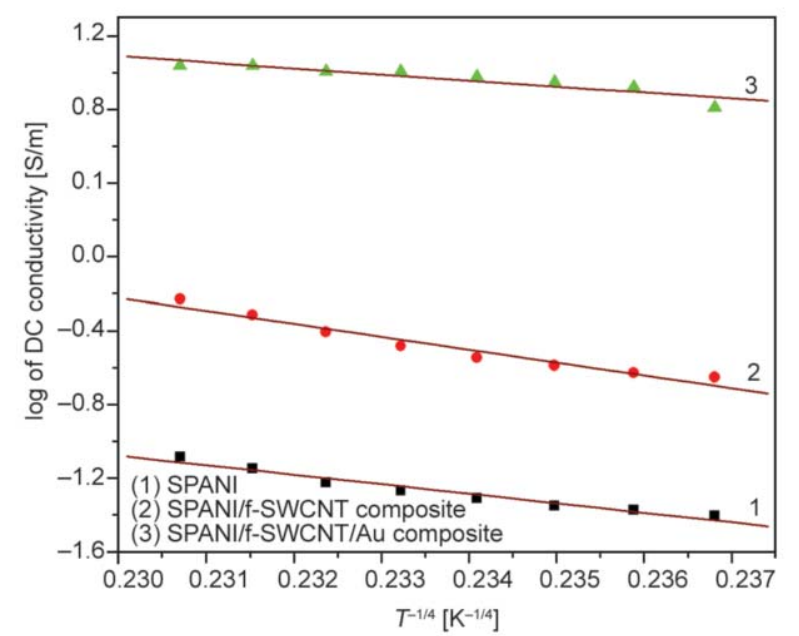

Figure 11. Variation of log of DC conductivity of SPANI, SPANI/f-SWCNT binary composite and SPANI/ f-SWCNT/Au ternary composite with $T^{-1 / 4}\left[\mathrm{~K}^{-1 / 4}\right]$ satisfying VRH model with $\gamma=1 / 4$

semiconductor, and supports the band gap estimations from the absorption data plotted in Figure 7.

In order to understand the electrical conduction mechanism, the log of dc conductivity of all samples were plotted against $T^{-1 / 4}$, which resulted in a straight line indicating a three-dimensional variable range hopping (3D VRH) conduction mechanism of Mott (Figure 11) [25]. The estimated values of Mott temperature were $4.38 \cdot 10^{6}, 1.38 \cdot 10^{7}$ and $1.08 \cdot 10^{6} \mathrm{~K}$ for SPANI, SPANI/f-SWCNT binary composite and SPANI/f-SWCNT/Au ternary composite, respectively. The significant decrease of Mott temperature in the ternary composite indicates a less disordered system, which is due to the presence of $\mathrm{f}-\mathrm{SWCNT} / \mathrm{Au}$ nanohybrid fillers in the sample. A similar decrease in Mott temperature due to introduction of Au nanoparticles into PANI has been observed previously by other researchers $[23,25]$.

\section{Conclusions}

In the present study, we have shown a simple wet chemical route to synthesize a 3-phase nanocomposite of SWCNT/Au nanohybrid fibers embedded in sulfonated polyaniline with excellent solution and electrical properties. The sulfonated composite is water soluble which can be very useful for its further processing. The synthesized nanocomposite has been characterized for its structural, thermal, electrical and optical properties. Optical absorption spectroscopy was used to estimate the band gaps which showed reduction I band gap as a function of incorporation of SWCNT and SWCNT/Au nanohybrids fillers. The DC electrical conductivity measurements showed significant increase in the electrical conductivity of the ternary composite compared to those of both SPANI and SPANI/SWCNT binary composite, in good agreement with the measured band gaps. The enhanced conductivity of the water soluble composite may find applications in electromagnetic interference (EMI) shielding [26], energy storage etc. But such a three-component polymer composite can primarily be utilized as an electrode material in biosensors [27] because of the excellent electrochemical properties of polyaniline and its composites, as well as for their long-term environmental stability. Further Au has a great affinity to attach biomolecules.

\section{Acknowledgements}

The authors are indebted to the TEQIP- I scheme of the Government of India for providing the laboratory and instrumental facilities at National Institute of Technology Durgapur, India. We also express thanks to the MHRD funded Centre of Excellence in Advanced Materials at NIT Durgapur for providing laboratory support.

\section{References}

[1] Li X-G., Feng H., Huang M-R., Gu G-L., Moloney M. G.: Ultrasensitive $\mathrm{Pb}(\mathrm{II})$ potentiometric sensor based on copolyaniline nanoparticles in a plasticizer-free membrane with a long lifetime. Analytical Chemistry, 84, 134-140 (2012). DOI: $10.1021 / \mathrm{ac} 2028886$

[2] Li X-G., Huang M-R., Lu Y-Q., Zhu M-F.: Synthesis and properties of processible copolymer microparticles from chloroanilines and aniline. Journal of Materials Chemistry, 15, 1343-1352 (2005).

DOI: $10.1039 / \mathrm{B} 412587 \mathrm{H}$

[3] Meriga V., Valligata S., Sundaresan S., Cahill C., Dhanak V. R., Chakraborty A. K.: Optical, electrical, and electrochemical properties of graphene based water soluble polyaniline composites. Journal of Applied Polymer Science, 132, 42766/1-42766/9 (2015). DOI: $10.1002 /$ app.42766

[4] De Volder M. F. L., Tawfick S. H., Baughman R. H., Hart A. J.: Carbon nanotubes: Present and future commercial applications. Science, 339, 535-539 (2013). DOI: $10.1126 /$ science. 1222453

[5] Chakraborty A. K., Coleman K. S., Dhanak V. R.: The electronic fine structure of 4-nitrophenyl functionalized single-walled carbon nanotubes. Nanotechnology, 20, 155704/1-155704/6 (2009). DOI: $10.1088 / 0957-4484 / 20 / 15 / 155704$

[6] Kar P., Choudhury A.: Carboxylic acid functionalized multi-walled carbon nanotube doped polyaniline for chloroform sensors. Sensors and Actuators B: Chemical, 183, 25-33 (2013).

DOI: $10.1016 / \mathrm{j} . \mathrm{snb} .2013 .03 .093$ 
[7] Lafuente E., Callejas M. A., Sainz R., Benito A. M., Maser W. K., Sanjuán M. L., Saurel D., de Teresa J. M., Martínez M. T.: The influence of single-walled carbon nanotube functionalization on the electronic properties of their polyaniline composites. Carbon, 46, 1909-1917 (2008). DOI: 10.1016/j.carbon.2008.07.039

[8] Kang M. S., Shin M. K., Ismail Y. A., Shin S. R., Kim S. I., Kim H., Lee H., Kim S. J.: The fabrication of polyaniline/single-walled carbon nanotube fibers containing a highly-oriented filler. Nanotechnology, 20, 085701/1085701/6 (2009).

DOI: 10.1088/0957-4484/20/8/085701

[9] Dhand C., Solanki P. R., Datta M., Malhotra B. D.: Polyaniline/single-walled carbon nanotubes composite based triglyceride biosensor. Electroanalysis, 22, 2683 2693 (2010).

DOI: 10.1002/elan.201000269

[10] Wei X-L., Wang Y. Z., Long S. M., Bobeczko C., Epstein A. J.: Synthesis and physical properties of highly sulfonated polyaniline. Journal of the American Chemical Society, 118, 2545-2555 (1996).

DOI: $10.1021 /$ ja952277i

[11] Agrawalla R. K., Paul R., Sahoo P. K. Chakraborty A. K., Mitra A. K.: A facile synthesis of a novel optoelectric material: A nanocomposite of SWCNT/ZnO nanostructures embedded in sulfonated polyaniline. International Journal of Smart and Nano Materials, 5, 180-193 (2014). DOI: $10.1080 / 19475411.2014 .937471$

[12] Santhosh P., Gopalan A., Lee K-P.: Gold nanoparticles dispersed polyaniline grafted multiwall carbon nanotubes as newer electrocatalysts: Preparation and performances for methanol oxidation. Journal of Catalysis, 238, 177-185 (2006).

DOI: $10.1016 /$ j.jcat.2005.12.014

[13] Feng X., Mao C., Yang G., Hou W., Zhu J-J.: Polyaniline/Au composite hollow spheres: Synthesis, characterization, and application to the detection of dopamine. Langmuir, 22, 4384-4389 (2006). DOI: $10.1021 / 1 \mathrm{a} 053403 \mathrm{r}$

[14] Wang Z., Yuan J., Li M., Han D., Zhang Y., Shen Y., Niu L., Ivaska A.: Electropolymerization and catalysis of well-dispersed polyaniline/carbon nanotube/gold composite. Journal of Electroanalytical Chemistry, 599, 121-126 (2007).

DOI: $10.1016 /$ j.jelechem.2006.09.021

[15] Chang Q., Kai Z., Chen X., Li M., Liu J.: Preparation of gold/polyaniline/multiwall carbon nanotube nanocomposites and application in ammonia gas detection. Journal of Materials Science, 43, 5861-5866 (2008). DOI: $10.1007 / \mathrm{s} 10853-008-2827-3$

[16] Lee K-P., Gopalan A. I., Santhosh P., Lee S. H., Nho Y. C.: Gamma radiation induced distribution of gold nanoparticles into carbon nanotube-polyaniline composite. Composites Science and Technology, 67, 811-816 (2007).

DOI: $\underline{10.1016 / \text { j.compscitech.2005.12.030 }}$
[17] Guo L., Peng Z.: One-pot synthesis of carbon nanotubepolyaniline-gold nanoparticle and carbon nanotube-gold nanoparticle composites by using aromatic amine chemistry. Langmuir, 24, 8971-8975 (2008).

DOI: $10.1021 / 1 \mathrm{a} 8010458$

[18] Rima P., Mitra A. K.: Synthesis and study of optical and electrical characteristics of single-wall carbon nanotube/gold nanohybrid. Journal of Nano Research, 17, 27-33 (2012).

DOI: 10.4028/www.scientific.net/JNanoR.17.27

[19] Ansari M. O., Khan M. M., Ansari S. A., Amal I., Lee J., Cho M. H.: pTSA doped conducting graphene/polyaniline nanocomposite fibers: Thermoelectric behavior and electrode analysis. Chemical Engineering Journal, 242, 155-161 (2014). DOI: $10.1016 /$ j.cej.2013.12.033

[20] Scolari M., Mews A., Fu N., Myalitsin A., Assmus T., Balasubramanian K., Burghard M., Kern K.: Surface enhanced Raman scattering of carbon nanotubes decorated by individual fluorescent gold particles. Journal of Physical Chemistry C, 112, 391-396 (2008). DOI: $10.1021 / \mathrm{jp} 076190 \mathrm{i}$

[21] Sumanasekera G. U., Allen J. L., Fang S. L., Loper A. L., Rao A. M., Eklund P. C.: Electrochemical oxidation of single wall carbon nanotube bundles in sulfuric acid. Journal of Physical Chemistry B, 103, 4292-4297 (1999). DOI: $10.1021 /$ jp984362t

[22] Tauc J.: Optical properties and electronic structure of amorphous Ge and Si. Materials Research Bulletin, 3, 37-46 (1968).

DOI: $10.1016 / 0025-5408(68) 90023-8$

[23] Gupta K., Jana P. C., Meikap A. K.: Electrical transport and optical properties of the composite of polyaniline nanorod with gold. Solid State Sciences, 14, 324-329 (2012).

DOI: $10.1016 /$ j.solidstatesciences.2011.12.003

[24] Mott N. F., Davis E. A.: Electronic processes in noncrystalline materials. Oxford University Press, New York (1979).

[25] Chakraborty G., Ghatak S., Meikap A. K., Woods T., Babu R., Blau W. J.: Characterization and electrical transport properties of polyaniline and multiwall carbon nanotube composites. Journal of Polymer Science Part B: Polymer Physics, 48, 1767-1775 (2010).

DOI: $10.1002 /$ polb.22042

[26] Saini P., Choudhary V., Singh B. P., Mathur R. B., Dhawan S. K.: Polyaniline-MWCNT nanocomposites for microwave absorption and EMI shielding. Materials Chemistry and Physics, 113, 919-926 (2009). DOI: 10.1016/j.matchemphys.2008.08.065

[27] Dhand C., Dwivedi N., Mishra S., Solanki P. R., Mayandi V., Beuerman R. W., Ramarishna S., Lakshminarayanan R., Malhotra B. D.: Polyaniline-based biosensors. Nanobiosensors in Disease Diagnosis, 4, 2546 (2015) DOI: $10.2147 /$ NDD.S64841 